\title{
Influência dos teores de aveia e de gordura nas características tecnológicas e funcionais de bolos
}

\author{
Influence of oat and fat levels in technological and functional characteristics of cakes
}

Luiz Carlos GUTKOSKI ${ }^{1 \star}$ Débora Marli de Freitas TEIXEIRA² ${ }^{2}$ Angelise DURIGON², Ana Gabriela GANZER ${ }^{2}$, Telma Elita BERTOLIN ${ }^{3}$, Luciane Maria COLLA ${ }^{2}$

\begin{abstract}
Resumo
O presente trabalho objetivou estudar o emprego de aveia e de gordura nas características tecnológicas e funcionais de bolos. No estudo foram utilizados os ingredientes flocos e farinha de aveia (Avena sativa L.) do cultivar IAC 7 (30\% de flocos grossos tostados e $70 \%$ de farinha), previamente selecionados com base na composição química, farinha de trigo, açúcar, sal, fermento químico, emulsificante, leite em pó, ovo inteiro, sorbitol e aroma de baunilha. $\mathrm{O}$ experimento foi realizado em delineamento composto central rotacional aplicável à metodologia de superfície de resposta (MSR), sendo os teores de aveia (flocos, farinha) e gordura estabelecidos como variáveis independentes. Os resultados de densidade da massa, viscosidade da massa, composição química, energia metabolizável, volume específico, atividade de água, características internas, porosidade e cor foram tratados por análise de regressão múltipla, obtendo-se modelo matemático de segunda ordem com os termos lineares, quadráticos e de interação significativos. A aveia pode ser utilizada na elaboração de bolos de valor calórico reduzido e como fonte de fibras alimentares atendendo às características de alimento funcional. A densidade da massa variou com a quantidade de gordura da formulação, enquanto que a viscosidade, com a concentração de aveia. A porosidade do miolo foi influenciada pelas concentrações de aveia e de gordura utilizadas nas formulações. Com a elevação do teor de aveia e a diminuição de gordura nas formulações ocorreu um aumento da área, diâmetro e perímetro médios dos alvéolos.

Palavras-chave: Avena sativa L.; farinha mista; alimento funcional; fibra; valor calórico.
\end{abstract}

\begin{abstract}
The present work aimed to study the use of oat and fat in the technological and functional characteristics of cakes. The ingredients used were oat flakes and flour (Avena sativa L.), of the cultivar IAC 7 (30\% of toasted thick flakes and $70 \%$ of flour), selected based on the chemical composition, wheat flour, sugar, salt, baking powder, emulsifier, powdered milk, whole egg, sorbitol, and vanilla flavor. The experiment was carried out using a central composite rotational design applied to the response surface methodology (RSM). The levels of oat (flakes, flour) and fat were established as independent variables. The results of dough density, dough viscosity, chemical composition, metabolizable energy, specific volume, water activity, internal characteristics, porosity, and color were analyzed by multiple regression; and the significant linear, quadratic, and interaction terms were used in the second order mathematical model. Oat can be used for preparing cakes with reduced caloric value, sources of dietary fiber, which are characteristics of functional foods. The dough density varied with the amount of fat added in the formulation while the viscosity varied with the oatmeal concentration. The porosity of the cake crumbs was influenced by the oatmeal and fat concentrations used in the formulations. With the increase of the oat level and decrease of fat in the formulations, the average area, diameter, and perimeter of the alveoli increased.
\end{abstract}

Keywords: Avena sativa L.; composite flour; functional food; fiber; caloric value.

\section{Introdução}

A aveia (Avena sativa L.) destaca-se entre os cereais por fornecer aporte energético e nutricional equilibrado, conter em sua composição química aminoácidos, ácidos graxos, vitaminas e sais minerais indispensáveis ao organismo humano e, principalmente, pela composição de fibras alimentares (WEBER; GUTKOSKI; ELIAS, 2002).

O uso de produtos de aveia como ingredientes na panificação é recomendável devido às suas propriedades funcionais tecnológicas, como a retenção de umidade, retardando com isso o envelhecimento de bolos. O uso de aveia melhora os teores de proteínas, fibra alimentar, bem como permite o aumento da variedade de produtos elaborados (GUTKOSKI et al., 2007).

Em adição aos efeitos físicos favoráveis, a aveia tem a capacidade de estabilizar os componentes lipídicos (CALDWELL et al., 1991) e de atuar como substituto de gordura (SEABRA; ZAPATA, 2002). Porém, a remoção total da gordura pode alterar o flavor, a textura, a leveza, o corpo e a suavidade do produto (KHALIL, 1998). A função da gordura é atrair ar para a massa durante a mistura. Quando o ar começa a se expandir, absorve cristais de gordura para a interface ar-água e assim forma-se a interface gordura-água;

${ }^{1}$ Faculdade de Agronomia e Medicina Veterinária, Universidade de Passo Fundo - UPF, CEP 99001-970, Passo Fundo - RS, Brasil, E-mail: gutkoski@upf.br

${ }^{2}$ Engenharia de Alimentos, Faculdade de Engenharia e Arquitetura, Universidade de Passo Fundo - UPF, CEP 99001-970, Passo Fundo - RS, Brasil

${ }^{3}$ Biologia, Instituto de Ciências Biológicas da Universidade de Passo Fundo - UPF, CEP 99001-970, Passo Fundo - RS, Brasil

${ }^{*}$ A quem a correspondência deve ser enviada 
ambas as fases se unem para a expansão das bolhas (KOCER, 2007). Se não houver algum agente estabilizador, essas bolhas de ar se rompem e migram para a superfície da massa. Essa função de estabilização é exercida pela fração cristalina da gordura. Além disso, quando a gordura é misturada à farinha, ocorre a dispersão de forma irregular, interrompendo a continuidade da cadeia de glúten que se forma quando proteínas começam a ser hidratadas, criando assim áreas frágeis na estrutura (CAUVAIN, 1997).

Na elaboração de bolos é necessário selecionar matérias-primas adequadas, empregar formulação balanceada e procedimentos de mistura e cozimento de acordo com o tipo de produto que se quer produzir. Na mistura dos ingredientes deve ser obtida uma dispersão homogênea, com máxima incorporação de ar e mínimo desenvolvimento do glúten (CALDWELL et al., 1991). A massa de bolo é uma emulsão de gordura em água complexa, composta por bolhas e uma mistura de ovo-açúcar-água-gordura na qual são dispersas partículas de farinha de trigo. Durante o assamento, aumento de temperatura, pressão de vapor de água e taxa de formação de dióxido de carbono resultam na expansão da massa. Aumento adicional de temperatura causa gelatinização do amido e coagulação de proteínas (KOCER, 2007).

Os bolos apresentam-se em diferentes formatos, sabores e textura, variando com a formulação e com o método empregado na elaboração. Estes produtos requerem o uso de farinha de trigo brando e permitem o emprego de farinhas sucedâneas de trigo como a aveia e outros alimentos funcionais (EL-DASH; GERMANI, 1994).

O trabalho objetivou estudar características tecnológicas e funcionais de bolos elaborados com o emprego de aveia e gordura através das análises de densidade e viscosidade da massa, composição química, energia metabolizável, volume específico, atividade de água, características internas, porosidade e cor.

\section{Material e métodos}

\subsection{Matéria-prima}

Foram utilizados os ingredientes flocos grossos tostados e farinha de aveia (Avena sativa L.) do cultivar IAC 7, previamente selecionados, apresentando $19,28 \%$ de proteína bruta, 7,04\% de lipídios, $10,00 \%$ de umidade, 10,08\% de fibra alimentar total, $2,28 \%$ de cinzas e $51,32 \%$ de carboidratos. Os flocos de aveia se diferenciam da farinha pela sua maior granulometria. A farinha de trigo foi produzida pelo moinho Companhia Brasileira de Moagem (Tio Hugo, RS), apresentando 10,95\% de proteína bruta, $0,63 \%$ de cinzas, $13,40 \%$ de umidade, 230 segundos de número de queda e $160 \mathrm{~W} \times 10^{-4}$ de força geral de glúten. Os ingredientes gordura vegetal hidrogenada marca Bunge Pro (Bunge, Esteio, RS) emulsificante estearoil-2-lactil lactato de sódio (SSL) (Granotec do Brasil, Curitiba, PR), fermento químico Biomica (Novo Hamburgo, RS), aroma de baunilha em pó (Duas Rodas Industrial, Jaraguá do Sul, SC), sorbitol (Bond Carneiro, Curitiba, PR), ovo inteiro, açúcar cristal, sal e leite em pó desnatado foram adquiridos no comércio local.

\subsection{Elaboração dos bolos de aveia}

Os bolos foram elaborados no laboratório de Panificação do Centro de Pesquisa em Alimentação (Cepa) da Universidade de Passo Fundo (UPF) a partir de formulação base definida em ensaios preliminares. Os bolos foram elaborados pelo emprego de farinha de trigo (100\%), açúcar (85\%), sal (1\%), fermento químico $(4 \%)$, emulsificante $(0,3 \%)$, leite em pó $(6 \%)$, ovo inteiro (4\%), sorbitol $(2,5 \%)$ e aroma de baunilha $(0,9 \%)$. As quantidades de aveia (30\% de flocos grossos tostados e 70\% de farinha pela substituição de parte da farinha de trigo) e de gordura vegetal hidrogenada (base farinha) foram adicionadas de acordo com o delineamento experimental. Os ingredientes foram pesados em balança semianalítica, a massa preparada em batedeira KitchenAid modelo K5SS (Heavy Duty, Michigan, EUA). Os ingredientes açúcar, sal, gordura, ovos e sorbitol foram misturados em velocidade 6, durante 7 minutos. A seguir, foram incorporados água e os demais ingredientes secos (farinha de trigo, fermento químico, emulsificante, leite em pó e aroma de baunilha), misturados e homogeneizados em velocidade 6, durante 3 minutos. Porções de $280 \mathrm{~g}$ de massa foram acondicionadas em formas de papel Kraft (com dimensões de 18,0 × 4,0 $\times$ $7,7 \mathrm{~cm}$ ) e assadas à temperatura de $180^{\circ} \mathrm{C}$ durante 30 minutos, em forno experimental modelo QA 226 (Labor Instruments Works, Hungria).

\subsection{Delineamento experimental}

As quantidades de aveia e de gordura foram estabelecidas como variáveis independentes, estudadas em cinco níveis codificados em $-\alpha,-1,0,+1 \mathrm{e}+\alpha$, de acordo com um delineamento experimental composto central rotacional $2^{2}$, visando a análise dos resultados pela metodologia de superfície de resposta (BOX; DRAPER, 1987). Os níveis de variação e as variáveis independentes estão apresentados na Tabela 1. No delineamento experimental foram realizados onze experimentos, com três repetições no ponto central (Tabela 2). As quantidades de aveia e de gordura foram definidas a partir da revisão de literatura e de testes experimentais realizados no laboratório de Panificação do Cepa/UPF.

\subsection{Análises}

\section{Composição química}

As determinações de umidade, proteínas, lipídios e cinzas dos bolos foram realizadas de acordo com os procedimentos da AACC (1995), métodos números 44-15 A, 46-12, 30-20 e 08-12, respectivamente. A determinação de fibra alimentar total foi realizada segundo procedimento da AOAC (2005), método número 991.43. O teor de carboidratos (excluindo-se a fração fibra alimentar total) foi estimado por diferença, diminuindo-se de 100 o somatório de proteínas, lipídios, cinzas, umidade e fibra alimentar total. As análises de composição química foram realizadas em duplicata e os valores expressos em porcentagem.

Tabela 1. Variáveis independentes e níveis de variação utilizados nos ensaios.

\begin{tabular}{lccccc}
\hline \multicolumn{1}{c}{ Variáveis independentes } & \multicolumn{5}{c}{ Níveis de variação } \\
\hline & $-a$ & -1 & 0 & +1 & $+\alpha$ \\
Aveia: flocos (30\%) e farinha (70\%) & 0,0 & 10 & 35 & 60 & 70 \\
Gordura (\%) & 8,7 & 15 & 30 & 45 & 51,2 \\
\hline
\end{tabular}


Tabela 2. Delineamento experimental composto central rotacional para duas variáveis.

\begin{tabular}{ccc}
\hline Experimento & Aveia (\%) & Gordura (\%) \\
\hline 1 & $10(-1)$ & $15(-1)$ \\
2 & $60(1)$ & $15(-1)$ \\
3 & $10(-1)$ & $45(1)$ \\
4 & $60(1)$ & $45(1)$ \\
5 & $0(-\alpha)$ & $30(0)$ \\
6 & $70(+\alpha)$ & $30(0)$ \\
7 & $35(0)$ & $8,7(-\alpha)$ \\
8 & $35(0)$ & $51,2(+\alpha)$ \\
9 & $35(0)$ & $30(0)$ \\
10 & $35(0)$ & $30(0)$ \\
11 & $35(0)$ & $30(0)$ \\
\hline
\end{tabular}

$a=1,414$ para $\mathrm{K}=2$ (duas variáveis independentes).

\section{Energia metabolizável}

A energia metabolizável dos bolos foi calculada a partir dos dados de composição centesimal aproximada, de acordo com a RDC n 360 do Ministério da Saúde (Brasil, 2003). No cálculo foram usados os fatores de conversão de $4 \mathrm{kcal}^{-1} \mathrm{~g}^{-1}$ para carboidratos e proteínas e de $9 \mathrm{kcal} . \mathrm{g}^{-1}$ para lipídios, e os valores expressos em kcal.g ${ }^{-1}$.

\section{Volume específico}

Para a determinação do volume específico, os bolos foram cortados ao meio, pesados e, em equipamento apropriado dotado de recipiente de volume conhecido e contendo sementes de painço, foi determinado o volume ocupado por cada pedaço de bolo nesse recipiente, através do deslocamento das sementes. $\mathrm{O}$ volume específico médio, expresso $\mathrm{em} \mathrm{cm}^{3} \cdot \mathrm{g}^{-1}$ foi obtido pela razão entre o volume e o peso de cada um dos pedaços de bolo.

\section{Determinação da atividade de água (aw)}

A determinação da atividade de água foi realizada pelo emprego do equipamento Testo, modelo 650, à temperatura constante $\left(22^{\circ} \mathrm{C} \pm 2\right)$, em duplicata.

\section{Características internas dos bolos}

As características internas dos bolos foram avaliadas após 4 horas do assamento, realizado de acordo com método no $10-90$ da $\mathrm{AACC}^{1}$, por quatro avaliadores treinados.

\section{Densidade e viscosidade da massa}

A densidade da massa crua foi determinada pela relação entre o peso $(\mathrm{g})$ e o volume $\left(\mathrm{cm}^{3}\right)$, realizado de acordo com Zambrano et al. (2005) em duplicata e os resultados expressos em $\mathrm{g}\left(\mathrm{cm}^{3}\right)^{-1}$. Amostras foram coletadas no final da mistura da massa realizada na batedeira KitchenAid, colocadas em recipiente cilíndrico tarado medindo $3,70 \mathrm{~cm}$ de diâmetro por $6,70 \mathrm{~cm}$ de altura.

A viscosidade da massa foi determinada no analisador rápido de viscosidade (RVA) (Newport Scientific Instruments,
Sidney, Austrália) pela suspensão de $10 \mathrm{~g}$ de massa em $25 \mathrm{~mL}$. O recipiente de alumínio contendo a amostra foi colocado no equipamento e submetido ao ensaio utilizando o tempo de cinco minutos, temperatura de $50{ }^{\circ} \mathrm{C}$ e velocidade de $160 \mathrm{rpm}$, sendo determinada a viscosidade expressa em centipoise $(\mathrm{cP})$ e realizados os ensaios em duplicata.

\section{Porosidade do miolo}

Amostras de fatias de bolo de 1,5 cm de espessura foram fotografadas pelo uso da câmera SCC-421P Samsung com placa de captura de imagens PV-BT878P+ e software TV Station V10.01a. As imagens com área central de 5,0 $\times 5,0 \mathrm{~cm}$ foram analisadas pelo emprego do software de imagens Image-Pro Plus 4.5 (Media Cybernetics Inc., EUA) e a porosidade determinada através dos parâmetros alveolares área $\left(\mathrm{mm}^{2}\right)$, diâmetro $(\mathrm{mm})$ e perímetro $(\mathrm{mm})$ médios, realizado de acordo com Esteller e Lannes (2005), em duplicata.

\section{Cor}

A cor da crosta e do miolo dos bolos foi determinada pelo uso do espectrofotômetro de reflectância difusa, modelo ColorQuest II Sphere (Hunter Associates Laboratory, Inc., Reston, EUA), com sensor ótico geométrico de esfera. $\mathrm{O}$ aparelho foi calibrado com cerâmica, realizando-se a leitura por reflexão e utilizando-se ângulo de observação de $10^{\circ}$, iluminante principal D65, iluminante secundário flu-branca fria e reflexão especular incluída (RSIN). No sistema Hunter de cor, corrigido pela CIELab, os valores L (luminosidade) flutuam entre zero (preto) e 100 (branco), os valores de a e b (coordenadas de cromaticidade) variam de $-\mathrm{a}$ (verde) até $+\mathrm{a}$ (vermelho), e -b (azul) até $+\mathrm{b}$ (amarelo). As amostras foram colocadas sobre o sensor ótico de $2,54 \mathrm{~mm}$, realizando-se três leituras em posições diferentes.

\section{Análise estatística}

As médias dos resultados das variáveis respostas de cada um dos onze experimentos foram tratadas por análise de regressão múltipla para verificar a possibilidade de descrever os efeitos das variáveis independentes com modelos matemáticos de segunda ordem e realizar a análise das superfícies de resposta. Nos modelos ajustados foi observada a significância da regressão pelo teste F e pelo coeficiente de determinação (BARROS NETO; SCARMINIO; BRUNS, 1995). O processamento dos dados foi realizado com o programa SAS ${ }^{\circledast}$, versão 6.12 (SAS INSTITUTE, North Carolina, EUA, 1985) e as Figuras elaboradas no programa Statistica. Os resultados de composição química e valor calórico dos experimentos selecionados foram analisados estatisticamente pela determinação de média e desvio padrão.

\section{Resultados e discussão}

A partir dos dados experimentais das variáveis respostas estudadas estabeleceram-se os modelos de regressão ajustados em função das condições experimentais empregadas, sendo significativos os apresentados na Tabela 3. A adequabilidade dos modelos pode ser verificada pelos coeficientes de determinação, que explicam entre 0,78 e 0,98 da variância total das respostas e pela ausência da falta de ajuste dos resíduos. Em razão desses resultados, os 
modelos podem ser usados para predizer o comportamento das respostas dentro do intervalo de variação estudado.

\subsection{Densidade e viscosidade da massa}

Na Figura 1, representa-se, na forma de gráficos de superfície de resposta, a variação da densidade (a) e da viscosidade (b) da massa dos bolos em função das concentrações de aveia e gordura empregadas nas formulações.

A densidade da massa dos bolos de aveia aumentou com a redução do teor gordura, variando entre 0,85 e 1,16 g.mL $\mathrm{m}^{-1}$. A formulação com $51,2 \%$ de gordura permitiu obter uma massa com menor densidade $\left(0,85\right.$ g. $\left.\mathrm{mL}^{-1}\right)$. Em contrapartida, a formulação com 8,7\% de gordura apresentou maior densidade $\left(1,16\right.$ g.mL $\left.{ }^{-1}\right)$. Os resultados de densidade apresentaram relação com a quantidade de gordura utilizada nas formulações. Bath, Shelke e Hoseney (1992) verificaram que a retenção do ar e dos gases da fermentação reflete na densidade e na viscosidade da massa. As formulações com substitutos de gordura produzem massas com densidades significativamente maiores e menor incorporação de ar quando comparadas às massas contendo gordura.

A viscosidade da massa sofreu grande interferência da quantidade de aveia utilizada nas formulações, porém a influência da gordura foi consideravelmente menor. Os valores de viscosidade das massas dos bolos variaram entre 150 e $650 \mathrm{cP}$, sendo verificado o maior valor de viscosidade na formulação com $70 \%$ de aveia e $30 \%$ de gordura, ocorrido devido à granulometria de flocos de aveia. Com o aumento de aveia nas formulações também ocorreu incremento da quantidade de fibra alimentar solúvel, entretanto, como a massa estava crua e no ensaio a temperatura utilizada foi abaixo da necessária para a gelatinização do amido, sua influência na viscosidade foi relativamente pequena.

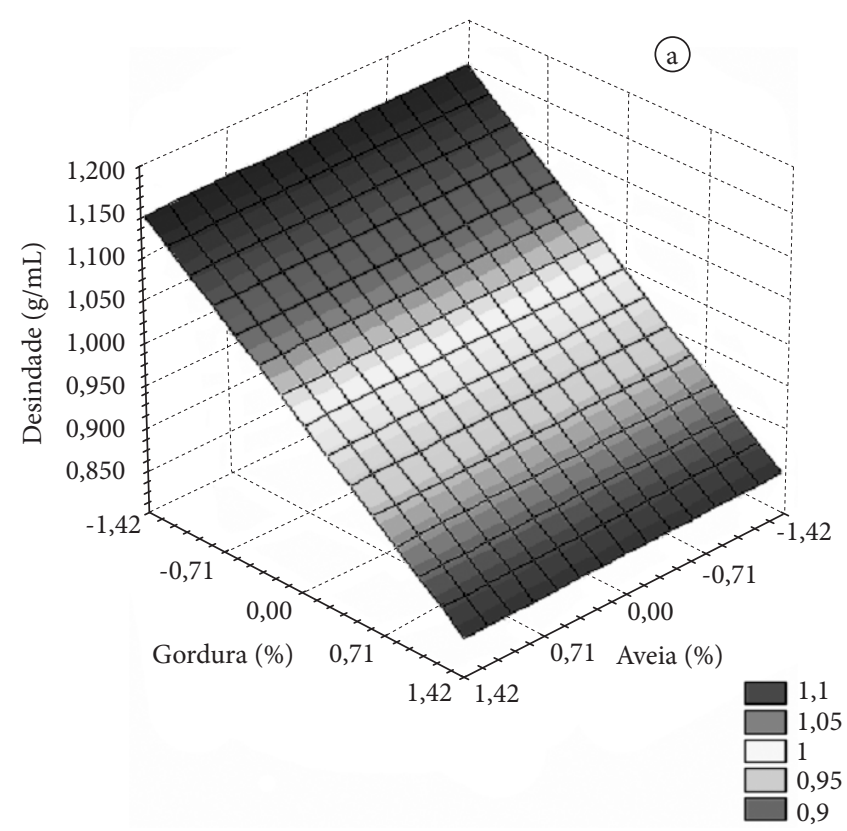

\subsection{Volume específico, características internas e atividade de água dos bolos}

A Figura 2 representa o diagrama de superfície correspondente ao modelo ajustado que estabelece a variação do volume específico (a) e das características internas (b) em função das concentrações de aveia e de gordura nas formulações.

Os valores de volume específico obtidos estão dentro da faixa de $1,6 \mathrm{~mL} \cdot \mathrm{g}^{-1}$ (60\% de aveia e $45 \%$ de gordura) a $2,2 \mathrm{~mL} \cdot \mathrm{g}^{-1}$ ( $10 \%$ de aveia e $15 \%$ de gordura) sendo menor na formulação com maior quantidade de gordura provavelmente pelos altos teores utilizados nas formulações. O volume específico sofreu influência linear negativa da quantidade de gordura utilizada nas formulações. Não houve efeito do teor de aveia adicionado. Zambrano et al. (2005) não verificaram influência no volume específico de bolos ao empregar as gomas guar e xantana como substitutos de gordura.

As características internas dos bolos sofreram influências linear e quadrática da quantidade de gordura adicionada com maiores pontuações nas formulações com baixos teores de gordura. Os teores de gordura superiores (acima de 30\%) são excessivos, trazendo prejuízos para as características dos bolos, o ideal se encontra entre 8,7 e $30 \%$.

Cauvain, 1987, afirma que a aeração proporcionada pela gordura é de vital importância no processo de fabricação dos bolos. A estrutura é formada durante o estágio de batimento, quando minúsculas bolhas de ar são incorporadas à massa. Essas bolhas se expandem quando a temperatura aumenta e, quando a massa endurece, explodem e formam a estrutura porosa tradicional. Assim, se não houver algum agente estabilizador, essas bolhas de ar se rompem e migram para a superfície da massa. Essa função de estabilização é exercida pela fração cristalina

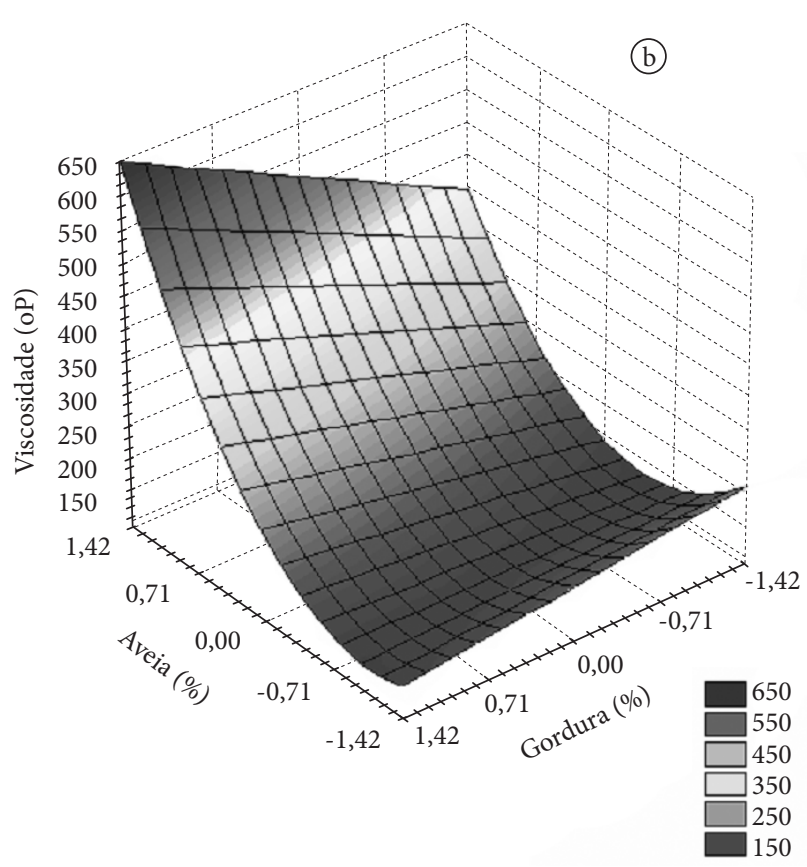

Figura 1. Efeito das concentrações de aveia (\%) e gordura (\%) na densidade (a) e na viscosidade (b) da massa utilizada na elaboração dos bolos. 
da gordura. Além disso, quando a gordura é misturada com a farinha, ocorre a dispersão de forma irregular, interrompendo a continuidade da cadeia de glúten que se forma quando as proteínas começam a se hidratar, criando assim áreas frágeis na estrutura. $\mathrm{O}$ resultado é que o produto se torna mais fácil de mastigar e, em geral, quanto mais gordura menos compacto ele fica. Isto ocorre até o emprego de uma quantidade limite de gordura e, a partir desse ponto, a gordura passa a atuar como compactante, reduzindo o volume do produto, comportamento verificado no presente trabalho.

A $\mathrm{a}_{\mathrm{w}}$ indica a quantidade de água disponível para realizar o movimento molecular e suas transformações e promover o crescimento microbiano no produto. A atividade de água média dos bolos foi de 0,88 , sendo o maior valor $(0,90)$ encontrado na formulação com $0 \%$ de aveia e $30 \%$ de gordura, e o menor valor $(0,86)$ foi observado na formulação com $60 \%$ de aveia e $15 \%$ de gordura. Segundo Jardim e Germer, 1997, valores acima de 0,80 a 0,88 favorecem o desenvolvimento de bolores e leveduras, respectivamente. A atividade de água sofreu influência linear e quadrática da quantidade de aveia e influência quadrática da quantidade de gordura (Tabela 3 ).

\subsection{Cor da crosta e porosidade do miolo}

A Figura 3 representa o diagrama de superfície correspondente ao modelo ajustado que estabelece a variação da intensidade da cor vermelha da crosta (a), assim como a variação da luminosidade do miolo (b) dos bolos em função das concentrações de aveia e de gordura nas formulações.

Dentre os parâmetros de cor analisados, apresentaram diferenças significativas os parâmetros luminosidade do miolo $\left(L^{\star}\right)$ e intensidade da cor vermelha da crosta $\left(+a^{*}\right)$. Maiores valores de $+\mathrm{a}^{*}$ indicam coloração mais avermelhada da crosta, sendo esta característica observada nas formulações com $45 \mathrm{e}$ $30 \%$ de gordura. Os valores mais baixos de $+\mathrm{a}^{\star}$ foram observados nas formulações com 8,7 e $15 \%$ de gordura. A intensidade da cor vermelha na crosta dos bolos não sofreu interferência da quantidade

Tabela 3. Modelos de regressão, significância e coeficientes de determinação para as respostas densidade (Den), viscosidade (Vis), atividade de água $\left(\mathrm{A}_{\mathrm{w}}\right)$, volume específico (VEsp), características internas (CarInt), luminosidade do miolo ( $\mathrm{L}^{\star}$ miolo), intensidade de vermelho da crosta ( $\mathrm{a}^{*}$ crosta $)$, área média $\left(\mathrm{A}_{\text {média }}\right)$, diâmetro médio $\left(\mathrm{D}_{\text {médio }}\right)$, e perímetro médio $\left(\mathrm{P}_{\text {médio }}\right)$ alveolares das formulações de bolos.

\begin{tabular}{lllc}
\hline Resposta & \multicolumn{1}{c}{ Modelo } & Prob $>\mathrm{F}$ & $\mathrm{R}^{2}$ \\
\hline Den & $0,99-0,10 \mathrm{X}_{2}$ & 0,0001 & 0,9854 \\
Vis & $184,53+124,12 \mathrm{X}_{1}+31,96 \mathrm{X}_{2}+81,01 \mathrm{X}_{1}^{2}+41,00 \mathrm{X}_{1} \mathrm{X}_{2}$ & 0,0013 & 0,9295 \\
$\mathrm{~A}_{\mathrm{w}}$ & $0,87-0,0128 \mathrm{X}_{1}+0,0082 \mathrm{X}_{1}{ }^{2}+0,0035 \mathrm{X}_{2}^{2}$ & 0,0001 & 0,94444 \\
VEsp & $1,89-0,21 \mathrm{X}_{2}$ & 0,0001 & 0,8371 \\
CarInt & $92,81-9,36 \mathrm{X}_{2}-5,84 \mathrm{X}_{2}^{2}$ & 0,0004 & 0,8628 \\
$\mathrm{~L}^{*}$ miolo & $69,13-4,88 \mathrm{X}_{1}-0,89 \mathrm{X}_{2}-0,85 \mathrm{X}_{2}{ }^{2}$ & 0,0001 & 0,9589 \\
$\mathrm{a}^{*}$ crosta & $17,03+3,24 \mathrm{X}_{2}-2,16 \mathrm{X}_{2}^{2}$ & 0,0022 & 0,7826 \\
$\mathrm{~A}_{\text {média }}$ & $10,29+1,30 \mathrm{X}_{1}-0,56 \mathrm{X}_{2}-0,42 \mathrm{X}_{1}^{2}$ & 0,0015 & 0,8744 \\
$\mathrm{D}_{\text {médio }}$ & $2,62+0,10 \mathrm{X}_{1}-0,10 \mathrm{X}_{2}-0,04 \mathrm{X}_{1}^{2}$ & 0,8754 \\
$\mathrm{P}_{\text {médio }}$ & $7,20+0,80 \mathrm{X}_{1}-0,29 \mathrm{X}_{2}-0,28 \mathrm{X}_{1}{ }^{2}$ & 0,0015 & 0,9072 \\
\hline
\end{tabular}

Modelos de regressão elaborados com o emprego de variáveis codificadas; $\mathrm{X}_{1}=$ quantidade de aveia (\%); $\mathrm{X}_{2}=$ quantidade de gordura (\%).
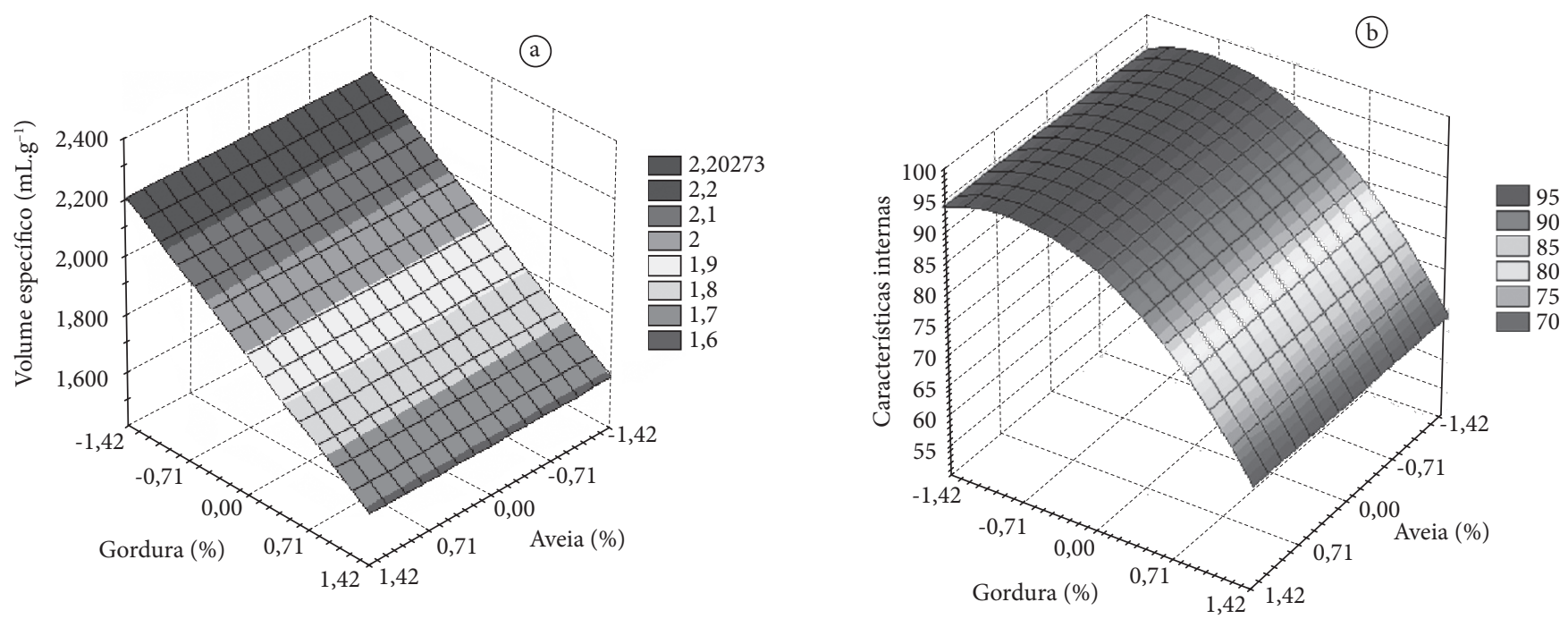

Figura 2. Efeito das concentrações de aveia (\%) e gordura (\%) no volume específico (a) e nas características internas (b) das formulações de bolos. 
de aveia empregada nas formulações, o que é justificado pela leitura ter sido realizada na crosta dos bolos (Tabela 3).

Valores mais altos de luminosidade do miolo indicam maior reflectância da luz traduzindo-se em bolos com coloração mais clara, fato observado nas formulações com menores quantidades de aveia (Figura 3b). A luminosidade do miolo dos bolos estudados teve influência linear tanto da quantidade de aveia quanto de gordura empregadas, ambas de forma negativa.

Na Figura 4, representa-se, na forma de gráficos de superfície de resposta, a variação dos parâmetros área (a) e diâmetro (b) médio alveolares dos bolos em função das concentrações de aveia e gordura nas formulações estudadas.

Dentre os parâmetros alveolares analisados apresentaram diferenças significativas os parâmetros área média, diâmetro médio e perímetro médio. Os valores mínimos para área, diâmetro e perímetro dos bolos analisados são os mesmos, devido ao limite de detecção do software de imagem. As diferenças aparecem para os valores máximos e médios, porém somente foram significativas as equações de regressão dos valores médios (Tabela 3). A quantidade e o volume dos alvéolos estão diretamente relacionados com as formulações estudadas.

A área média alveolar das formulações analisadas sofreu influência linear e quadrática da quantidade de aveia empregada e linear da quantidade de gordura. Os maiores valores de área média foram verificados nas formulações com elevadas quantidades de aveia e reduzidos teores de gordura.

O diâmetro médio sofreu influência tanto da quantidade de aveia quanto da quantidade de gordura empregadas nas formulações, sendo os maiores valores de diâmetro médio encontrados nas formulações contendo 35 e $60 \%$ de aveia e 8,7 e
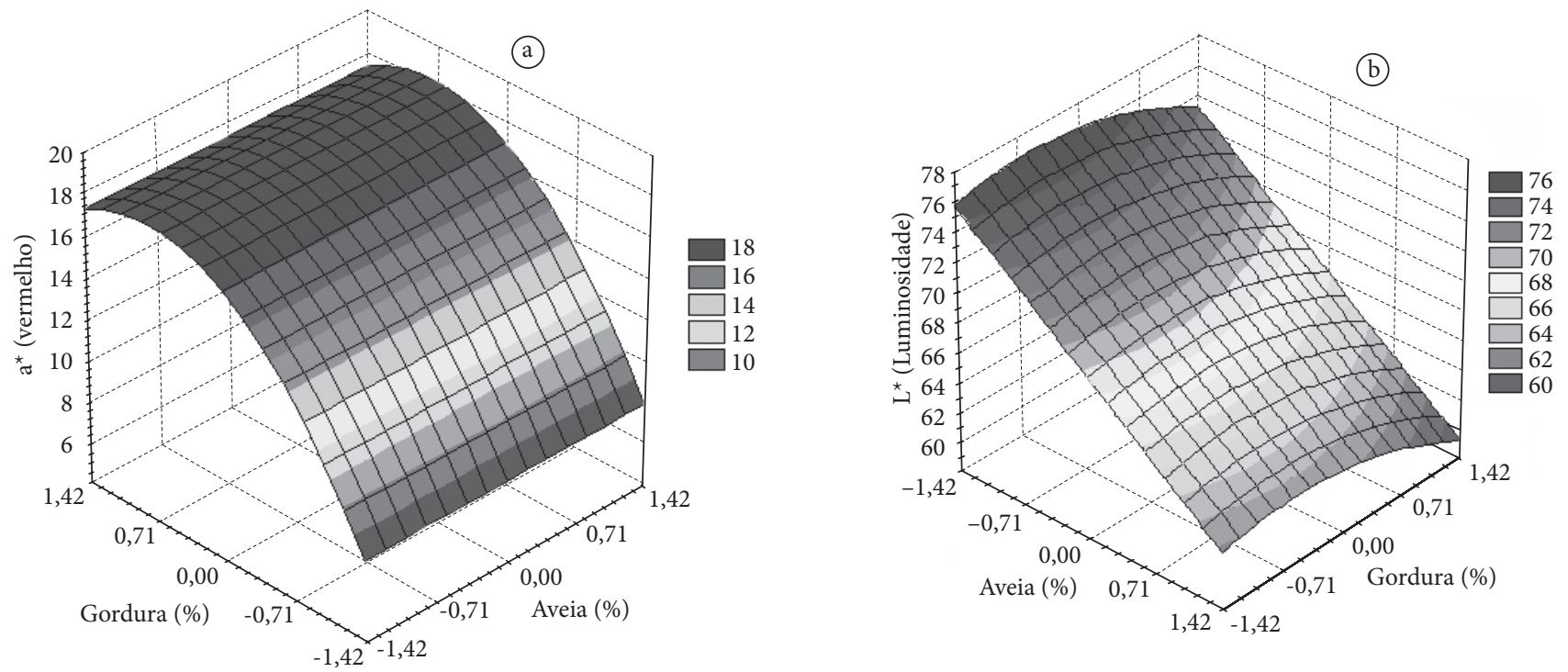

Figura 3. Efeito das concentrações de aveia (\%) e gordura (\%) na intensidade da cor vermelha da crosta (a) e luminosidade do miolo (b) das formulações dos bolos.
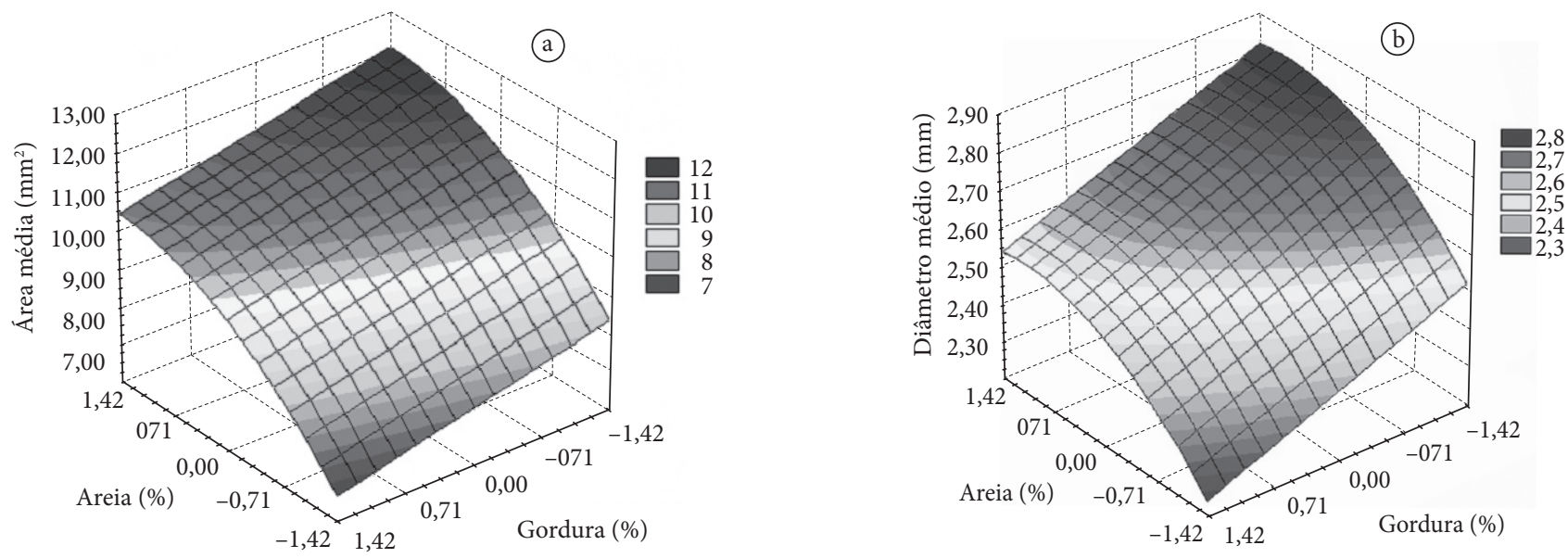

Figura 4. Efeito das concentrações de aveia (\%) e de gordura (\%) nos parâmetros área (a) e diâmetro (b) médio alveolares das formulações de bolos. 
$15 \%$ de gordura $\left(\mathrm{D}_{\text {médio }}=2,77\right.$ e 2,78 mm). Quanto mais elevada a quantidade de aveia utilizada nas formulações e reduzida a quantidade de gordura, maior o valor encontrado para o diâmetro médio. O perímetro médio apresentou comportamento similar ao verificado em área e diâmetro médio alveolar.

O emprego de sistemas de análise de imagem, que unem a câmera de captura de imagens ao computador e que apresentam como princípio a separação de imagens dentro de áreas pretas e brancas contra um segundo plano, permite avaliar com precisão parâmetros para a fixação de identidade e qualidade de produtos de panificação (ESTELLER; LANNES, 2005).

\subsection{Composição química de tratamentos selecionados}

Os resultados das análises de umidade, proteínas, lipídios, fibra alimentar total, cinzas, carboidratos e valor calórico total dos bolos selecionados (formulações 5, 6, 7 e 8) estão apresentados na Tabela 4. Os pontos selecionados foram os axiais por apresentar as quantidades extremas de aveia (formulações 5 e 6 ) e quantidades extremas de gordura (formulações 7 e 8 ) com valores médios da segunda variável independente estudada.

O maior valor de proteínas (7,89\%) foi verificado na formulação com $70 \%$ de aveia e $30 \%$ de gordura, com aumento de $25 \%$ em relação à formulação sem adição de aveia (formulação 5). A concentração de proteína do grão de aveia varia entre $12 \mathrm{e}$ $24 \%$, sendo que, das frações consumíveis, os maiores teores encontram-se no farelo e no endosperma, correspondendo em torno de 33 e $60 \%$ do peso do grão (DANIEL, 2006).

Os lipídios representam a fração mais calórica dos constituintes presentes nos alimentos, sendo a quantidade presente avaliada com maior rigor, em especial quando se trata de alimentos funcionais ou de reduzido valor calórico. Nos alimentos têm-se procurado substituir os lipídios por constituintes que apresentem propriedades similares, porém com menor contribuição energética para o produto (ZAMBRANO et al., 2005). O maior valor de lipídios $(21,15 \%)$ foi encontrado na formulação com 35\% de aveia e 51,2\% de gordura. O teor de lipídios está relacionado com a quantidade de gordura adicionada, tendo pouca influência da quantidade de aveia adicionada (como pode ser visto pela pequena diferença observada entra as formulações 5 e 6 , com 0 e $70 \%$ de aveia, respectivamente). O grão de aveia possui entre 5 e $9 \%$ de lipídios, destacando-se os polinsaturados, importantes nutricionalmente pela composição em ácidos graxos essenciais (DANIEL, 2006).

As fibras alimentares consistem em qualquer material comestível de origem vegetal que não seja hidrolisado pelas enzimas endógenas do trato digestivo humano (DE FRANCISCO; DE SÁ, 2001). Esta definição inclui lignina, polissacarídeos estruturais e não estruturais, excetuando-se o amido. Pectinas, gomas, mucilagens, alginatos, beta-glucanas e algumas hemiceluloses são consideradas fibras solúveis, enquanto a lignina, a celulose e a maioria das hemiceluloses são fibras insolúveis (CÂNDIDO; CAMPOS, 1995). Estudos epidemiológicos correlacionando o elevado consumo de fibra alimentar e a menor incidência de determinadas doenças, como as cardiovasculares e o câncer de cólon, impulsionaram as pesquisas sobre a fibra alimentar. Várias doenças, como o câncer de cólon e do reto, câncer de mama, diabetes, aterosclerose, apendicite, doença de Crohn, síndrome do intestino irritável, hemorroidas e diverticulite, têm sido relacionadas com uma baixa ingestão de fibras alimentares (HEATON, 1992). A formulação com $70 \%$ de aveia e $30 \%$ de gordura apresentou $2,78 \%$ de fibra alimentar total, representando um aumento de $30 \%$ em relação à formulação sem adição de aveia. Para que o bolo elaborado possa ser denominado fonte de fibra, a legislação brasileira exige que ele apresente em sua composição no mínimo 3 g $100 \mathrm{~g}^{-1}$ de fibra alimentar total, o que poderia ser obtido com substituição parcial da farinha pelo farelo de aveia, sem alterar as proporções dos constituintes estudados.

Com relação ao valor calórico, as formulações com 8,7 e 51,2\% de gordura apresentaram 295,48 e 366,15 kcal, respectivamente. A redução deste constituinte indica a possibilidade de elaboração de bolo com reduzido teor de gordura. Para que ocorra a redução de calorias é necessário que haja diminuição no teor de algum nutriente energético, podendo ser carboidratos, lipídios ou proteínas.

Na Figura 5 está representado o efeito das concentrações de aveia e de gordura na estrutura de miolo dos bolos (da esquerda para a direita, formulações 5, 7 e 8). O uso de produtos de aveia como ingredientes na panificação é recomendável devido às suas excelentes propriedades de retenção de umidade, retardando com isso o envelhecimento (WEBSTER, 1986). Em adição aos efeitos físicos favoráveis, a aveia tem a capacidade de estabilizar os componentes lipídicos. Outra razão para seu uso é a melhoria nos teores de proteínas, fibra alimentar, bem como o aumento da variedade de bolos produzidos (CALDWELL, 1991; DANIEL, 2006).

Tabela 4. Umidade (U), proteínas (P), lipídios (L), fibra alimentar total (FT), cinzas (C), carboidratos (CHO) e valor calórico total (VCT) de formulações de bolos com diferentes quantidades de aveia $(\mathrm{A})$ e de gordura $(\mathrm{G})$.

\begin{tabular}{|c|c|c|c|c|c|c|c|c|c|}
\hline \multirow[t]{2}{*}{$\mathrm{n}^{\circ}$} & \multicolumn{2}{|c|}{$\begin{array}{c}\text { Variáveis } \\
\text { independentes }\end{array}$} & \multicolumn{7}{|c|}{ Determinações $^{1}$} \\
\hline & $\begin{array}{c}\text { A } \\
(\%)\end{array}$ & $\begin{array}{c}G \\
(\%)\end{array}$ & $\begin{array}{c}\mathrm{U} \\
(\%)\end{array}$ & $\begin{array}{c}\mathrm{P} \\
(\%)\end{array}$ & $\begin{array}{c}\mathrm{L} \\
(\%)\end{array}$ & $\begin{array}{c}\text { FT } \\
(\%)\end{array}$ & $\begin{array}{c}\mathrm{C} \\
(\%)\end{array}$ & $\begin{array}{c}\mathrm{CHO} \\
(\%)\end{array}$ & $\begin{array}{l}\text { VCT } \\
\text { (kcal) }\end{array}$ \\
\hline 5 & 0 & 30 & $32,64 \pm 0,01$ & $5,98 \pm 0,14$ & $13,26 \pm 0,13$ & $1,95 \pm 0,00$ & $1,82 \pm 0,00$ & $44,35 \pm 0,05$ & $320,66 \pm 2,1$ \\
\hline 6 & 70 & 30 & $26,42 \pm 0,90$ & $7,89 \pm 0,00$ & $15,10 \pm 0,73$ & $2,78 \pm 0,00$ & $2,26 \pm 0,01$ & $45,55 \pm 0,54$ & $349,66 \pm 4,0$ \\
\hline 7 & 35 & 8,7 & $28,95 \pm 0,90$ & $7,28 \pm 0,00$ & $6,64 \pm 2,06$ & $2,54 \pm 0,10$ & $2,94 \pm 0,00$ & $51,65 \pm 1,28$ & $295,48 \pm 6,0$ \\
\hline 8 & 35 & 51,2 & $30,52 \pm 0,00$ & $6,31 \pm 0,01$ & $21,15 \pm 2,46$ & $1,95 \pm 0,15$ & $2,43 \pm 0,00$ & $37,64 \pm 1,62$ & $366,15 \pm 9,2$ \\
\hline
\end{tabular}

${ }^{1}$ Os valores representam a média de duas determinações \pm desvio padrão 

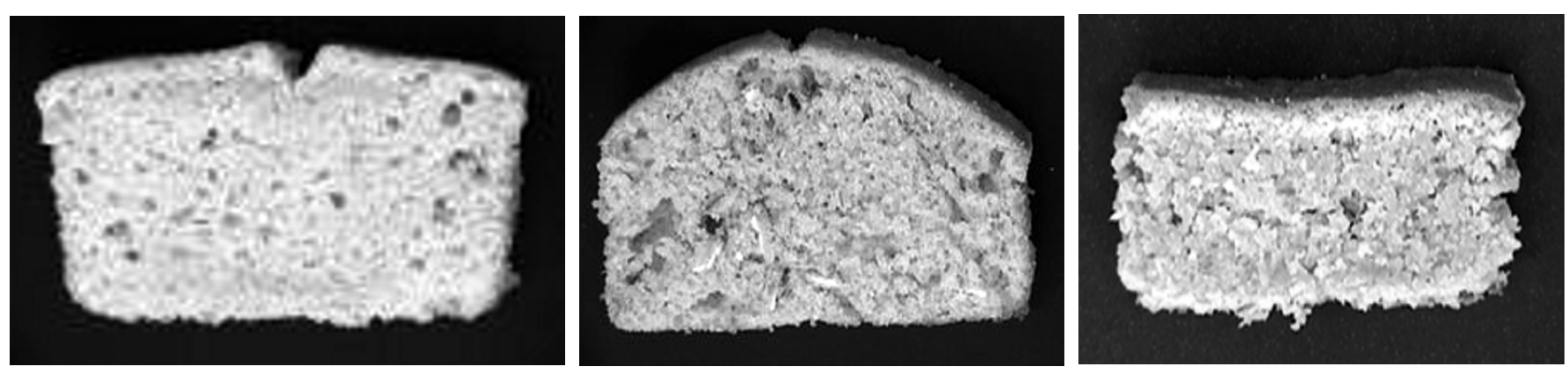

Figura 5. Efeito das concentrações de aveia (\%) e gordura (\%) na estrutura de miolo dos bolos (da esquerda para a direita, formulações 5, 7 e 8).

\section{Conclusões}

A aveia pode ser utilizada na elaboração de bolos de valor calórico reduzido e como fonte de fibras alimentares atendendo às características de alimento funcional. A densidade da massa variou com a quantidade de gordura da formulação, enquanto que a viscosidade, com a concentração de aveia. A porosidade do miolo foi influenciada pelas concentrações de aveia e de gordura utilizadas nas formulações. Com a elevação do teor de aveia e diminuição de gordura nas formulações, ocorreu um aumento da área, do diâmetro e do perímetro médios dos alvéolos.

\section{Agradecimentos}

Ao MCT/CNPq pelo apoio financeiro e bolsa de produtividade em pesquisa. Ao programa PIBIC/UPF pelas bolsas de iniciação científica. À empresa SL Alimentos pelo material experimental.

\section{Referências bibliográficas}

AMERICAN ASSOCIATION OF CEREAL CHEMISTS. Approved methods of the American Association of Cereal Chemists. 9 ed. Saint Paul: AACC, 1995. v. 2.

ASSOCIATION OF OFFICIAL ANALYTICAL CHEMISTRY. Official methods of analysis of the Association of Official Analytical Chemistry. 18 ed. Arlington, Washington: AOAC, 2005.

BARROS NETO, B.; SCARMINIO, I. S.; BRUNS, R. E. Planejamento e otimização de experimentos. 2 ed. Campinas: Editora da Unicamp, 1995. 299 p.

BATH, D. E.; SHELKE, K.; HOSENEY, R. C. Fat replacers in high-ratio layer cakes. Cereal Foods World, v. 37, n. 7, p. 495-500, 1992.

BOX, G. P.; DRAPER, N. R. Empirical model-building and response surfaces. New York: J. Wiley, Sons, 1987. 669 p.

BRASIL. Ministério da Saúde. Agência Nacional de Vigilância Sanitária. Resolução-RDC no 360, de 23 de dezembro de 2003. Dispõe sobre o regulamento técnico de rotulagem nutricional de alimentos embalados. Diário Oficial da República Federativa do Brasil, Brasília, 17 de dezembro de 2003.

CÂNDIDO, L. M. B.; CAMPOS, A. M. Alimentos para fins especiais: dietéticos. São Paulo: Varela, 1995.

CALDWELL, E. F. et al. Hot cereals. In: FAST, R. B.; CALDWELL, E. F. (Ed). Breakfast cereals. Saint Paul: American Association of Cereal Chemists, 1991. p. 243-272.

CAUVAIN, S. Let them eat cake especially if it's low fat. Food Flav Ingred Packag Process, v. 9, n. 8, p. 37-39, 1987.
DANIEL, A. P. Fracionamento a seco da farinha de aveia e modificação química da fração rica em amido. Ciência e Tecnologia de Alimentos, v. 26, n. 4, p. 936-943, 2006.

DE FRANCISCO, A.; DE SÁ, R. M. Beta-glucanas: localização, propriedades e utilização. In: LAJOLO, F. M. et al. (Ed.). Fibra dietética en Iberoamérica: tecnología y salud. São Paulo: Varela, 2001.

EL-DASH, A.; GERMANI, R. Tecnologia de farinhas mistas. Brasília: Embrapa, 1994.

ESTELLER, M. S.; LANNES, S. C. S. Parâmetros complementares para fixação de identidade e qualidade de produtos panificados. Ciência e Tecnologia de Alimentos, v. 25, n. 4, p. 802-806, 2005.

GUTKOSKI, L. C. et al. Desenvolvimento de barras de cereais à base de aveia com alto teor de fibra alimentar. Ciência e Tecnologia de Alimentos, v. 27, n. 2, p. 787-792, 2007.

HEATON, K. W. Dietary fiber in the prevention and treatment of gastrointestinal disorders. In. SCHWEIZER, T. F.; EDUARDS, C. A. (Ed.). Dietary Fiber: a component of food. Suttgard: Sring Verlag, 1992. p. 260-263.

JARDIM, D. C. P; GERMER, S. P. M. Atividade de água em alimentos. Campinas: Ital, 1997. 2-3 p.

KHALIL, A. H. The influence of carboydrate-based fat replacers with and without emulsifiers on the quality characteristics of lowfat cake. Plant Foods Human Nutrition, v. 52, n. 4, p. 299-313, 1998.

KOCER, D. et al. Buble and pore formation of the high-ratio cake formulation with polydextrose as a sugar and fat-replacer. Journal Food Engineering. v. 78, n. 3, p. 953-964, 2007.

SAS-SATATISTICAL ANALYSIS SYSTEM. User's guide: statistics. 5 ed. Cary, 1985. 956 p.

SEABRA, L. M. J. et al. Fécula de mandioca e farinha de aveia como substitutos de gordura na formulação de hambúrguer de carne ovina. Ciência e Tecnologia de Alimentos, v. 22, n. 3, p. 244-248, 2002.

WEBER, F. H.; GUTKOSKI, L. C.; ELIAS, M. C. Caracterização química de cariopses de aveia (Avena sativa, L.) da cultivar UPF 18. Ciência e Tecnologia de Alimentos, v. 22, n. 1, p. 39-44, 2002.

WEBSTER, F. Oat utilization: past, present and future. In: WEBSTER, F. (Ed). Oats: chemistry and technology. Saint Paul: American Association of Cereal Chemists, 1986. p. 413-426.

ZAMBRANO, F. et al. Efeito das gomas guar e xantana em bolos como substitutos de gordura. Brazilian Journal Food Technology, v. 8, n. 1, p. 63-71, 2005. 